\title{
Arbor
}

\section{La conjetura de Poincaré. Un problema de topología}

\section{María Teresa Lozano Imízcoz}

Arbor CLXXVIII, 704 (Agosto 2004), 691-707 pp.

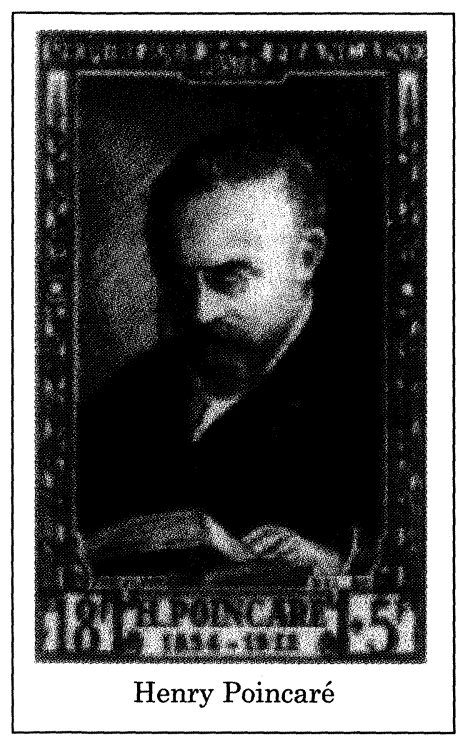

\section{Topología}

El gran matemático Henri Poincaré fue el creador de la Topología. Sólo un científico con su manera peculiar de pensar e investigar, novedosa para la época (su intuición geométrica era extraordinaria), podía imaginar y abstraer el contenido cualitativo de los objetos geométricos como él lo hizo.

La Topología, que Poincaré denominó "Analysis sitûs», es una rama de las Matemáticas que se ocupa de caracterizar algunas propiedades cualitativas de los objetos. Aquellas que permanecen tras una deformación continua sin roturas ni pegados. Es algo así como una geometría blanda. Esta original idea sólo es posible en una mente con una ex-

traordinaria capacidad de abstracción espacial. He aquí la definición dada por Poincaré en [Po3]:

El Analysis sitûs es la ciencia que nos hace conocer las propiedades cualitativas de las figuras geométricas no sólo en el espacio ordinario sino en espacios de más de tres dimensiones.

Mas adelante explica este grado de abstracción como la que realizamos en el arte de la geometría: razonar bien sobre figuras mal realizadas. Las proporciones de las figuras pueden ser alteradas, pero sus elementos no pueden ser trastocados y deben conservar su posición relativa. En otras 
palabras, las propiedades cuantitativas no son importantes, sino que se deben respetar las propiedades cualitativas, es decir precisamente aquellas de las que se ocupa el Analysis sitûs.

Los objetos de la categoría topológica son los espacios topológicos, y sus aplicaciones son las aplicaciones continuas. Las equivalencias, homeomorfismos, son aplicaciones biyectivas continuas con inversa continua. Podemos decir que en topología dos objetos (espacios topológicos) son iguales (homeomorfos) si uno se obtiene del otro por una deformación topológica (continua sin singularidades), llamada isotopía. Para un topólogo es lo mismo un poliedro sólido regular, un plato o una bola tridimensional. No es difícil imaginar la deformación sin rotura de cada uno de los dos primeros objetos anteriores en una bola si se suponen hechos de un material moldeable.

Figura 1. Objetos topológicamente iguales.
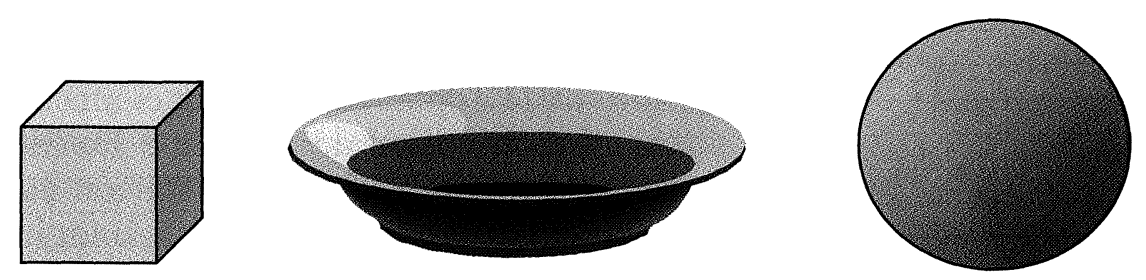

Uno de los principales problemas que se plantea en topología es la de reconocer cuando dos espacios son topológicamente iguales, porque a veces no es fácil encontrar una isotopía que transforme uno en el otro, aunque tal transformación exista. En estas deformaciones topológicas hay propiedades que permanecen, son aquellas de naturaleza topológica, y su estudio desemboca en la definición de los llamados invariantes.

Figura 2. Objetos topológicamente distintos.

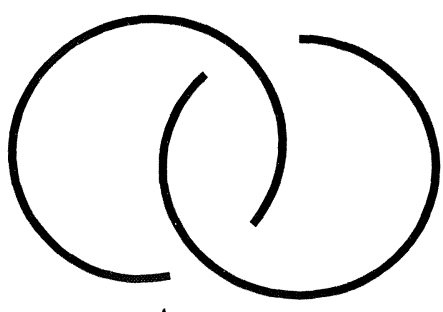

A

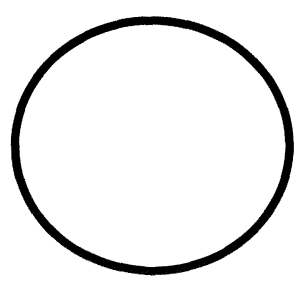

B 


\section{La conjetura de Poincaré. Un problema de topología}

En la Figura 2 se muestran dos espacios A y B. El espacio A está formado por dos circunferencias enlazadas y el espacio B es una circunferencia. Como en una deformación topológica el número de componentes es un invariante, se concluye que A y B no son espacios (homeomorfos) topológicamente iguales. Hemos visto aquí un sencillo ejemplo de invariante numérico: número de componentes conexas.

Poincaré ideó interesantes invariantes algebraicos que forman el núcleo de la Topología Algebraica. Esta rama de la topología asocia a cada espacio topológico objetos algebraicos (grupos, anillos,...) que sean invariantes, es decir, que cumplan que dos espacios homeomorfos tienen asignados objetos algebraicos isomorfos. Así, si para dos espacios $\mathrm{X}$ e $\mathrm{Y}$ encontramos un invariante algebraico $G$ que asigna a $X$ el objeto algebraico $G(X)$ y a $Y$ el objeto algebraico $\mathrm{G}(\mathrm{Y})$, de manera que $\mathrm{G}(\mathrm{X})$ y $\mathrm{G}(\mathrm{Y})$ no son isomorfos, podemos asegurar que $\mathrm{X}$ e $\mathrm{Y}$ no son homeomorfos. Los invariantes sirven fundamentalmente para asegurar que dos espacios no son homemorfos.

Otro importante problema en topología es caracterizar un espacio concreto X. Es decir, encontrar un sistema S completo de invariantes de ese espacio $\mathrm{X}$ que permita asegurar que cualquier otro espacio con el mismo valor para los invariantes del sistema S, es homeomorfo a X.

Para entender estos conceptos y avanzar en el enunciado de la conjetura objeto de esta conferencia, analizamos con más detalle el concepto topológico de esfera ó como es una esfera vista por un topólogo.

La primera definición de esfera es de origen geométrico. La esfera de dimensión $n, S^{n}$, es el conjunto de vectores unitarios del espacio Euclideo de dimensión $n+1, E^{n+1}$.

$$
S^{n}=\left\{\left(x_{1}, x_{2}, \ldots, x_{n+1}\right) \mid \sum_{i=0}^{n+1} x_{i}^{2}=1\right\}
$$

Figura 3. Esferas

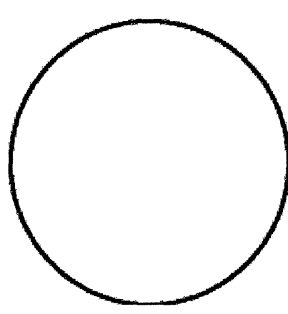

$S^{1}$

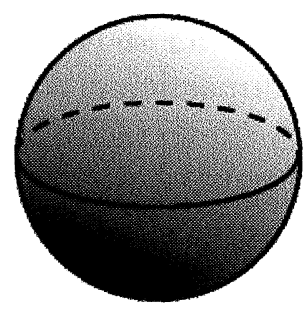

$S^{2}$

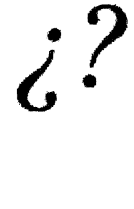

$S^{n}, n>2$ 
En la Figura 3 dibujamos la esfera $S^{n}$ como una figura geométrica. Así, la esfera $S^{1}$ es la circunferencia y la esfera $S^{2}$ es la superficie esférica común, pero tenemos dificultades cuando tratamos de pintar $S^{n}, n>2$. La razón es que no es posible incrustar toda la esfera $S^{n}, n>2$ en una porción del espacio tridimensional, que es nuestro campo visual, de la misma manera que no es posible incrustar (sin autointersecciones) la superficie esférica en una porción de plano.

Observamos que una esfera $S^{n}$ es un espacio con una notable propiedad local: cualquier punto, $x$, tiene un entorno, $U_{x}$, que es como un disco de dimensión $n, D^{n}$. En lenguaje topológico diremos que este entorno es homeomorfo a un disco (existe una aplicación biyectiva continua $f_{x}: U_{x} \rightarrow$ $D^{n}$ con inversa continua). Todo espacio con esta propiedad local recibe el nombre de variedad de dimensión $n$.

Considerar la esfera $S^{n}$ únicamente como variedad de dimensión $n$ es olvidar la estructura rígida que le da la geometría, conservando las demás propiedades cualitativas.

\section{Caracterización topológia de las esferas}

\subsection{La circunferencia $S^{1}$}

Desde el punto de vista topológico cualquier curva simple cerrada en el plano o en cualquier espacio de dimensión superior es una esfera $S^{1}$. Por tanto el número de componentes (uno) caracteriza la circunferencia o esfera de dimensión 1 entre todas las variedades de dimensión 1 compactas.

\subsection{La superficie esférica $S^{2}$}

En dimensión 2 el problema se complica, porque existen muchas variedades de dimensión 2 (superficies) conexas y compactas que no son homeomorfas a la esfera $S^{2}$, luego el invariante numérico dado por el número de componentes conexas, que era suficiente en dimensión 1 , no basta en dimensión 2 .

La caracterización de la esfera de dimensión 2 con una propiedad intrínseca que la distinga de las otras variedades de dimensión 2 (superficies), se consigue con un invariante algebraico definido y estudiado por 
La conjetura de Poincaré. Un problema de topología

Poincaré. Se trata del grupo fundamental o grupo de Poincaré, cuya definición recordamos a continuación.

Cada espacio topológico $X$ con un punto distinguido $x_{0}$, tiene asociado un grupo algebraico, $\pi_{1}\left(X, x_{0}\right)$, cuyos elementos son clases de equivalencia de caminos que empiezan y terminan en el punto $x_{0}$, a los que llamamos lazos. Dos lazos son equivalentes si se puede deformar uno en el otro de forma continua manteniendo fijos los extremos.

$$
\left.\pi_{1}\left(X, x_{0}\right)=\{\alpha] \mid \alpha:[0,1] \rightarrow X \text { continua, } \alpha(0)=\alpha(1)=x_{0}\right\}
$$

La composición de dos elementos $[\alpha],[\beta]$ del grupo es la clase que resulta de recorrer primero un camino $\alpha$, representante del primer elemento $[\alpha]$, y a continuación el camino $\beta$, representante del otro elemento $[\beta]$.

$$
\begin{array}{ccc}
\pi_{1}\left(X, x_{o}\right) \times \pi_{1}\left(X, x_{o}\right) & \rightarrow & \pi_{1}\left(X, x_{o}\right) \\
([\alpha][\beta] & \mapsto & {[\alpha * \beta]}
\end{array}
$$

El elemento neutro de esta composición es la clase del camino constante $c_{x_{0}}:[0,1] \rightarrow X, c_{x_{0}}(t)=x_{0}$, que envía todos los puntos del intervalo unidad al punto base $x_{0}$.

Figura 4. Caminos en superficies
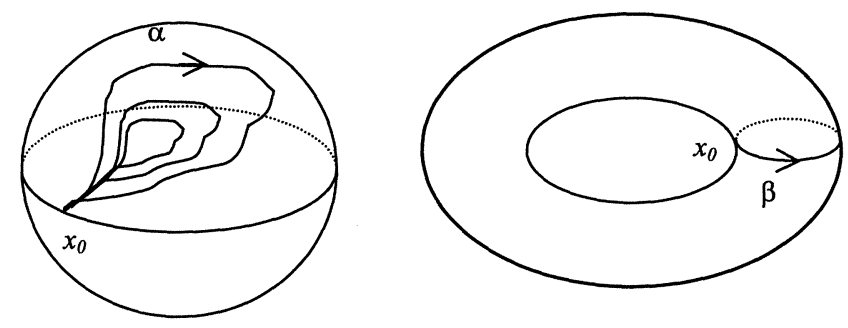

En la Figura 4 se ha dibujado un camino en una esfera de dimensión 2 y un camino $\beta$ en una superficie tórica. Es fácil ver que el camino $\alpha$ se puede deformar continuamente dentro de la superficie esférica al camino constante manteniendo en todo momento fijos los extremos en el punto $x_{0}$. Basta imaginar que la imagen del camino es un hilo elástico que se 
encoge durante la deformación. Esto sucede con todos los lazos basados en el punto $x_{0}$ que se pueden trazar en la superficie esférica. Sin embargo no es posible contraer a un punto el camino $\beta$ sin salirse de la superficie tórica o sin romper el camino. Este ejemplo ilustra el siguiente resultado:

R1: La esfera es la única superficie cerrada cuyo grupo fundamental es el grupo trivial:

$$
\pi_{1}\left(S^{2}, x_{0}\right)=\{0\}
$$

Es consecuencia de que cada camino cerrado se contrae a un punto, luego todos los lazos basados en $x_{0}$ son equivalentes al camino constante y por tanto su clase es la del elemento neutro.

R2: La esfera $S^{2}$ es la única superficie en la que cada camino cerrado es el borde de una superficie.

Cada camino cerrado es el borde de un disco inmerso (una porción de superficie), que es el área barrida durante la contracción a un punto.

Cada una de estas dos propiedades, $\mathrm{R} 1$ y R2, que son equivalentes, constituye una caracterización de la esfera $S^{2}$.

\subsection{La esfera tridimensional $S^{3}$ : Conjetura de Poincaré}

En 1900, Henri Poincaré, por analogía con la caracterización R2 de la esfera $S^{2}$, antes citada, escribió que también la esfera $S^{3}$ es la única variedad de dimensión 3 en la que toda curva cerrada bordea una superficie. [Po1].

Cuatro años mas tarde, en 1904, él mismo publicó en el quinto complemento al Analysis situs [Po2], un contraejemplo a esta supuesta caracterización. En el artículo describe una variedad de dimensión 3, hoy conocida como esfera homológica de Poincaré, en la que toda curva cerrada bordea una superficie, pero no es homeomorfa a la esfera tridimensional. De hecho esta variedad tiene un grupo fundamental de 120 elementos y su recubridor universal es la esfera $S^{3}$. Se puede definir como el conjunto de dodecaedros (o icosaedros) inscritos en una esfera bidimensional. Este ejemplo demuestra que la propiedad R2 que sirve para caracterizar la superficie esférica no es suficiente en dimensión 3. Además demuestra que en dimensión 3 el análogo a R2 es más débil que el análogo a R1, aunque ambas propiedades eran iguales en dimensión 2 . $\mathrm{El}$ artículo termina asegurando que la propiedad que caracteriza la esfe- 
La conjetura de Poincaré. Un problema de topología

ra tridimensional es la de tener grupo fundamental trivial (análoga a R1). La última frase de este escrito es:

Mais cette question nous entrainerait trop loin.

Nada más exacto. Su investigación ha sido objeto de estudio de muchos topólogos durante todo un siglo. Un enunciado preciso de la Conjetura de Poincaré es:

\section{Una variedad de dimensión 3 cerrada (compacta y sin borde) con grupo fundamental trivial es homeomorfa a la esfera tridimensional}

A primera vista puede parecer una sencilla afirmación, y es difícil imaginar un contraejemplo. Por eso han sido numerosos los matemáticos que a lo largo del siglo XX se han atrevido a presentar una demostración detallada, demostración que siempre ha resultado incompleta o errónea.

\subsection{Las esfera $S^{n}, n>3$. Conjetura de Poincaré generalizada}

Se observa fácilmente que existen variedades de dimensión $n(n>3)$ cerradas con grupo fundamental trivial que no son homeomorfas a la esfera. Por ejemplo, el producto cartesiano de esferas $S^{k} \times S^{n-k}$ donde $k>1$. Por tanto un enunciado como el de la conjetura de Poincaré en dimensión 3 es falso para dimensiones superiores a 3. La razón es que al aumentar la dimensión, aumenta la complejidad topológica de las variedades, y se hace necesario utilizar más invariantes para caracterizar un tipo concreto de variedad. La Topología Algebraica proporciona invariantes algebraicos que generalizan al grupo fundamental. Se trata de los grupos de homotopía $\pi_{i}$ cuyos elementos son clases de equivalencia de aplicaciones basadas de esferas basadas $\left(S^{i}, z_{0}\right)$ en la variedad basada $\left(M, m_{0}\right),(i=1$ es el grupo fundamental). Dos espacios X, Y se dice que son del mismo tipo de homotopía si tienen todos sus respectivos grupos de homotopía isomorfos: $\pi_{i}\left(X, x_{0}\right) \cong \pi_{i}\left(Y, y_{0}\right)$, para todo $i \in \mathbb{N}$. El enunciado de la conjetura en dimensión $n$ es:

\section{Una variedad de dimensión $n, n>3$, cerrada (compacta y sin borde) del mismo tipo de homotopía que la esfera $S^{n}$ es homeomorfa a la esfera $S^{n}$.}


La conjetura se resolvió primero para dimensión $n>4$. A partir de 1960 varios matemáticos probaron por diferentes métodos distintas versiones de esta Conjetura. En dimensión menor o igual que 3 es indiferente trabajar con variedades topológicas, combinatorias o diferenciables, pero esto no sucede en dimensión superior. La categoría de variedades utilizada y sus correspondientes métodos son lo que distingue las diversas demostraciones. Citemos a Smale [Sm], Stalling [St] y Wallace [W]. El hecho de que estos resultados contribuyeran a que Stephen Smale recibiera la medalla Fields en 1966, da idea de la importancia del tema.

La prueba en dimensión 4 fue obtenida veinte años mas tarde por $\mathrm{Mi}$ chael Freedman [F]. En el mismo artículo clasificó todas la 4-variedades cerradas y simplementes conexas. Por este y otros importantes resultados Michael Freedman recibió también una medalla Fields en 1986.

\section{Visualizando la esfera tridimensional}

Estamos acostumbrados a ver variedades de dimensión 1 y 2 dentro de nuestro espacio visual, lo que nos ayuda a estudiar geometría y topología en esas dimensiones. En particular tenemos una clara idea geométrica de lo que es una circunferencia y una superficie esférica, como vemos en la Figura 3. De esta idea geométrica podemos derivar una idea topológica permitiendo deformaciones continuas, al modo en que Dalí imaginó sus relojes blandos. Pero si ni siquiera podemos dibujar la esfera geométrica de dimensión 3 o más ¿cómo vamos a imaginar la esfera topológica $\mathrm{S}^{3}$ ?

Las siguientes ideas pretenden ayudar a pensar como un topólogo. En primer lugar nombramos algunos elementos notables de esfera geométrica $\left.S^{3}=\left\{x_{1}, x_{2}, x_{3}, x_{4}\right) \mid x_{1}^{2}+x_{2}^{2}+x_{3}^{2}+x_{4}^{2}=1\right\}$, por analogía con los de la superficie esférica.

$\begin{array}{ll}\text { Polo Norte: } & N=(0,0,0,1) \\ \text { Polo Sur: } & S=(0,0,0,-1) \\ \text { Hemisferio Norte: } & H_{N}=\left\{\left(x_{1}, x_{2}, x_{3}, x_{4}\right) \mid x_{4} \geq 0\right\} \\ \text { Hemisferio Sur: } & H_{S}=\left\{\left(x_{1}, x_{2}, x_{3}, x_{4}\right) \mid x_{4} \leq 0\right\} \\ \text { Ecuador: } & E=H_{N} \cap H_{S}=\left\{\left(x_{1}, x_{2}, x_{3}, x_{4}\right) \mid x_{4}=0\right\} \cong S^{2}\end{array}$


La conjetura de Poincaré. Un problema de topología

\subsection{Unión de dos bolas tridimensionales}

Es claro que cada hemisferio es una bola tridimensional puesto que

$$
x_{1}^{2}+x_{2}^{2}+x_{x}^{2}+x_{4}^{2}=1 \Leftrightarrow x_{1}^{2}+x_{2}^{2}+x_{x}^{2}=1-x_{4}^{2} \leq 1
$$

Ambos hemisferios tienen el ecuador como parte común, luego podemos representar $S^{3}$ como dos bolas tridimensionales pegadas por su esfera borde, es decir dos bolas identificadas por el borde mediante un homeomorfismo. Cualquier homeomorfismo (pegado) entre las esferas bordes de las dos bolas produce el mismo resultado. Figura 5.

Figura 5. Dos bolas identificadas por el borde

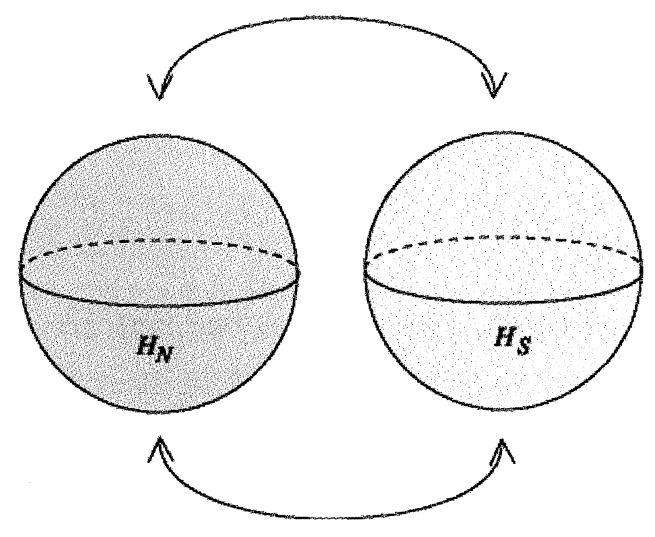

\subsection{Proyección estereográfica}

La proyección estereográfica desde el norte de la esfera al hiperplano ecuatorial

$$
\begin{aligned}
e: S^{3}-N & \rightarrow \\
\left(x_{1}, x_{2}, x_{3}, x_{4}\right) & \mapsto\left(\frac{x_{1}}{1-x_{4}}, \frac{x_{2}}{1-x_{4}}, \frac{x_{3}}{1-x_{4}}\right)
\end{aligned}
$$

es un homeomorfismo (aplicación biyectiva continua con inversa continua). Si identificamos la imagen con nuestro espacio ambiente, podemos pensar que la esfera tridimensional es su compactificación con un punto en el infinito. En esta representación el polo sur, S, es el origen de coor- 
denadas, el hemisferio sur es la bola unidad, cuyo borde, la esfera bidimensional unidad, es el ecuador, y el exterior de la bola unidad se corresponde con el hemisferio sur, entorno del punto del infinito que representa al polo norte. Figure 6 .

Figura 6. $R^{3} \cong S^{3}-N$

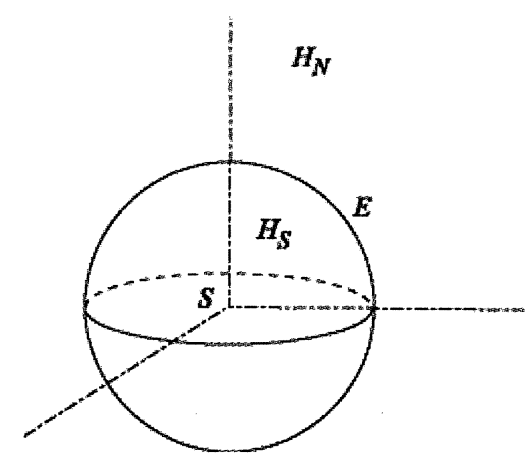

\subsection{Una bola con su borde identificado por reflexión en el ecuador}

Si partimos de la representación de $S^{3}$ como dos bolas pegadas por su esfera borde y hacemos primero la identificación de un disco, el resultado es homeomorfo a una bola en la que se debe identificar la esfera borde por reflexión en una línea. Ver Figura 7.

Figura 7. Una bola con autoidentificación en el borde

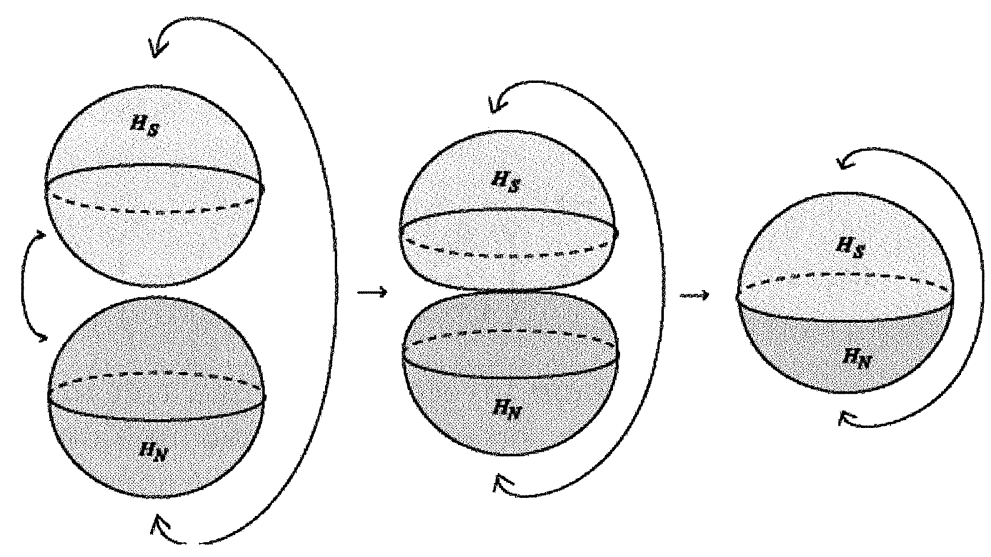




\section{La conjetura de Poincaré. Un problema de topología}

\subsection{Unión de dos toros sólidos}

En la representación anterior se considera la bola unidad en $R^{3}$ y se saca de su interior un entorno del eje Z. El resultado tiene dos componentes $A$ y $B$ en las que hay que realizar identificaciones. La componente $A$ es un cilindro en el que debe identificarse la base con la tapa, por tanto es un toro sólido. La componente $B$ es un toro sólido con una identificación en parte de su borde cuyo resultado sigue siendo un toro sólido. La identificación de los dos toros sólidos formados por A y B debe hacerse de manera que coincidan en $a$ que es meridiano de un toro y longitud del otro. Figura 8.

Figura 8. Dos toros sólidos $A$ y $B$
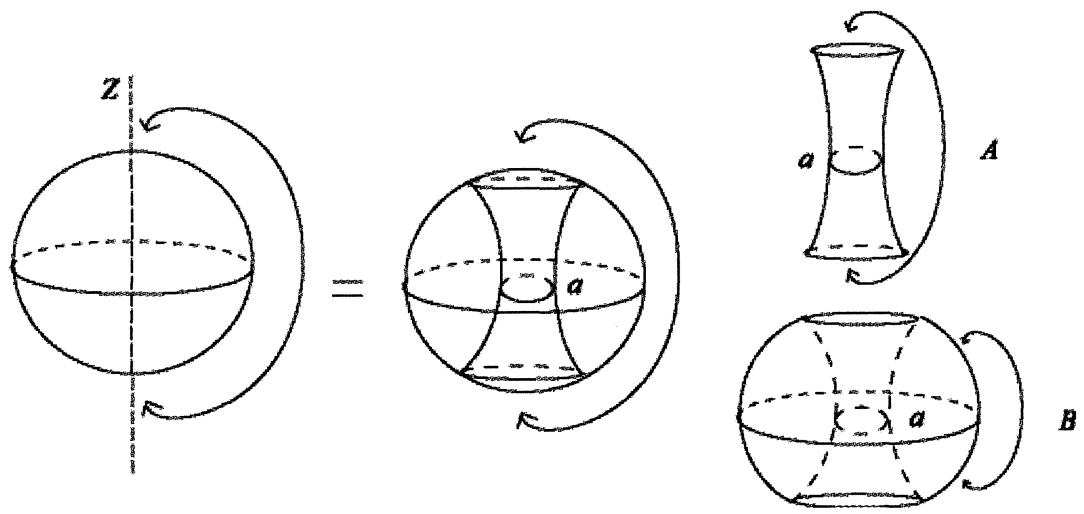

Se deduce de esta representación y de 3.2 que si se considera un toro sólido $\left(D^{2} \times S^{1}\right)$ no anudado en $R^{3}$, su complementario en $R^{3} \cup_{\infty} \cong S^{3}$ es también un toro sólido.

\subsection{Un cubo con cierto plegado de sus caras}

En la Figura 9 se representa un cubo que tiene en cada cara una linea central marcada con una etiqueta $a, b$ o $c$, que es paralela a uno de sus lados y que la divide en dos partes iguales, de manera que caras paralelas tienen líneas marcadas paralelas con la misma etiqueta, y dos líneas marcadas no tienen ningún punto en común. Si se identifica cada cara del cubo por plegado en la línea central marcada, el resultado es $S^{3}$, 
y las líneas marcadas forman el enlace conocido como anillos de Borromeo. Este enlace consta de tres circunferencias enlazadas en su conjunto, pero dos a dos desenganchadas. Si se elimina o se corta una cualquiera de ellas, las otras dos quedan desenlazadas.

Figura 9. Cubo con líneas marcadas

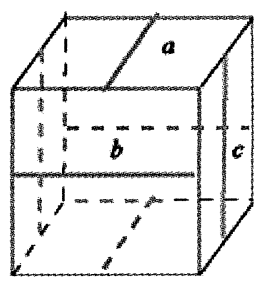

En la Figura 10 se ha dibujado el proceso de plegado en el cubo en varias etapas. En 1 se muestran las identificaciones a realizar. En 2 ya se han identificado las caras que tienen la línea central marcada con etique ta $a$, que han quedado en el interior del sólido. En 3 se ha identificado las caras laterales dejando las líneas marcadas con $c$ como una curva cerrada en el interior, a la vez las líneas marcadas con $b$ se han convertido en una curva cerrada en la esfera borde del sólido, que es una bola en la que se debe identificar el borde por reflexión en b, y eso (recordando 3.3) produce la esfera $S^{3}$. En 4 se ha dibujado sólo las curvas correspondientes a las aristas marcadas. Estas curvas forman los anillos de Borromeo.

Figura 10. Identificación en el cubo
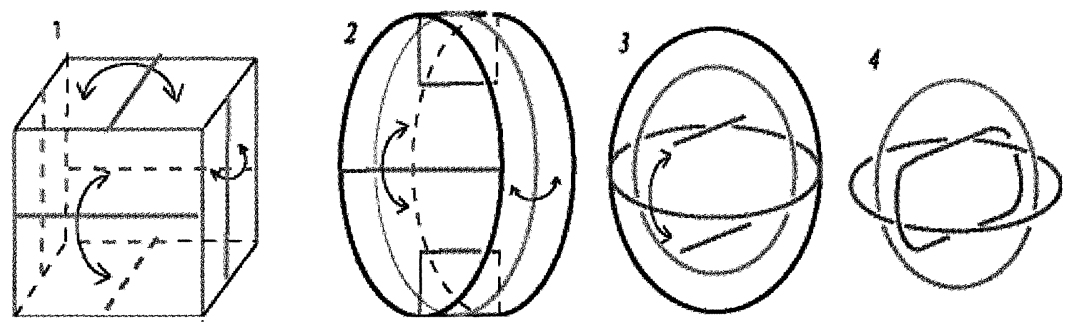

Esta representación de $S^{3}$ puede parecer sofisticada, pero tienen una justificación geométrica más profunda que vamos a esbozar. Podemos do- 
La conjetura de Poincaré. Un problema de topología

tar al cubo de la geometría euclídea que posee como cubo unidad del espacio euclideo tridimensional $E^{3}$. Cada plegado en una línea marcada se realiza por giro de $180^{\circ}$ en una recta, que es una isometría euclídea. Por tanto el cociente hereda una estructura geométrica "casi euclídea» conocida como estructura de orbifold euclídea, en la que todos los puntos tienen un entorno euclídeo salvo los puntos cociente de las líneas marcadas, los anillos de Borromeo, que constituyen la singularidad de la orbifold, y para los que el ángulo alrededor de un meridiano es, en este caso, de $180^{\circ}$ en lugar de $360^{\circ}$. Esta estructura de orbifold en $S^{3}$ se designa por $B_{2,2,2}$.

Figura 11. Un dodecaedro con aristas marcadas

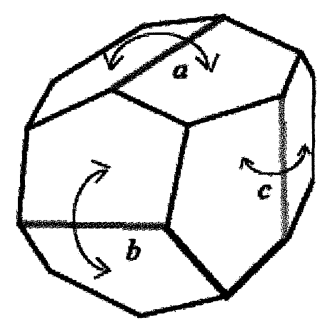

Un cubo con líneas marcadas como en la Figura 9 es combinatoriamente igual que un dodecaedro con aristas marcadas como en la Figura 11. Si se considera un dodecaedro regular en el espacio hiperbólico $H^{3}$ con ángulos diedrales de $90^{\circ}$ y se realiza el plegado indicado, el resultado es de nuevo $S^{3}$, pero con una estructura "casi hiperbólica» de orbifold $h i$ perbólica con los anillos de Borromeo como singularidad cuyo ángulo es de $90^{\circ}$. Esta estructura de orbifold en $S^{3}$ se designa por $B_{4,4,4}$

Por otra parte recordemos que $S^{3}$ soporta una estructura geométrica esférica, orbifold esférica sin ninguna singularidad.

Esta breve pincelada pretende ilustrar como en una misma variedad se pueden poner distintas estructuras rígidas geométricas de orbifold, hecho que enriquece notablemente el estudio de variedades.

\section{Buscando una solución a la conjetura de Poincaré}

Durante todo el siglo XX se han utilizado diversos métodos para resolver el problema. En numerosas ocasiones ha habido matemáticos que han presentado a la comunidad matemática una demostración. Pero en 
un tiempo más o menos breve, otros matemáticos han encontrado algún error o algún detalle no demostrado. Esto da idea de la complejidad del problema. Realmente no es difícil imaginar caminos de demostración, pero lo complicado es comprobar cada paso de la prueba.

\subsection{Un ejemplo}

En lo que sigue vamos a indicar, con un ilustrativo y simplificado ejemplo, los pasos a seguir para demostrar la conjetura.

En primer lugar fijamos un procedimiento para construir o visualizar todas las 3-variedades cerradas. Existen muchos procedimientos para hacerlo, generalizando los existentes para 2-variedades. Uno de ellos se deduce del Teorema 1.1 de [HLMW]: Toda 3-variedad cerrada es espacio recubridor de $S^{3}$ ramificada sobre los anillos de Borromeo con indices 1,2 y 4 .

Procedimiento. Toda 3-variedad cerrada es unión de un número finito de dodecaedros pegados por sus caras, de manera que cada arista pertenece a uno, dos ó cuatro dodecaedros.

Los datos en este caso son:

- $n$ el número de dodecaedros, que corresponde al número de hojas del espacio recubridor en el Teorema,

- los pegados de las caras, que vienen dados por la monodromía del espacio recubridor.

En segundo lugar, como desgraciadamente no conocemos un procedimiento que asocie a cada 3-variedad un único conjunto de datos (las 3-variedades cerradas no están totalmente clasificadas), hay que estudiar las transformaciones en los datos que no modifican la variedad que representan.

En nuestro caso hay que estudiar las jugadas que cambian el número de hojas o la monodromía, sin modificar el espacio recubridor.

En tercer lugar hay que relacionar el grupo fundamental de la variedad con los datos que la representan.

Para nosotros esto es factible algorítmicamente usando la relación existente entre espacios recubridores y grupos fundamentales.

A continuación, se establecen los datos que corresponden a una 3-variedad cerrada simplemente conexa.

Por último mediante el uso de jugadas que cambian los datos pero no cambian la 3-variedad, se trata de llegar a unos datos que correspondan a la 3-esfera. 


\section{La conjetura de Poincaré. Un problema de topología}

Como se puede deducir de este ejemplo (inconcluso hasta el momento), el camino es largo y con sutiles detalles a comprobar.

Nota: El camino esbozado, es una simplificación del método general aplicado a la representación de toda 3-variedad cerrada como orbifold hiperbó lica que cubre a la orbifold hiperbólica $B_{4,4,4}$ (con la 3-esfera como espacio subyacente y los anillos de Borromeo como singularidad de orden 4).

\subsection{Usando el flujo de Ricci}

En los últimos años, se han introducido en el estudio de las 3-variedades topológicas, métodos que utilizan estructuras Riemannianas.

Es bien conocido que cada superficie cerrada admite una estructura Riemanniana de curvatura constante, aquella que tiene su espacio recubridor universal. Las superficies orientables de género mayor o igual que 2 , y las no orientables de género mayor o igual que 3, tienen una estructura hiperbólica (curvatura contante negativa); el toro y la botella de Klein tienen una estructura Euclídea (curvatura 0); y la 2-esfera y el plano proyectivo tiene una estructura Riemanniana de curvatura constante positiva. Entonces se deduce que una superficie cerrada simplemente conexa con una estructura Riemanniana de curvatura constante positiva, es necesariamente la 2-esfera.

La analogía en dimensión 3, sugiere un camino de demostración de la conjetura de Poincaré. Se trata de demostrar que toda 3-variedad cerrada simplemente conexa posee una estructura Riemanniana de curvatura constante positiva, y por tanto es la 3-esfera.

El flujo de Ricci, fue ideado por Hamilton en $[\mathrm{H}]$ para variar de manera diferenciable la métrica en la variedad tendiendo hacia una estructura mas homogénea. Su definición es la siguiente. Sea M una 3-variedad cerrada. La familia de estructuras Riemannianas diferenciables, $\{g(t) \mid t \in[0, T)\}$, es flujo de Ricci si satisface $g^{\prime}(t)=2 \operatorname{Ric}(g(t))$, donde $\operatorname{Ric}(g(t))$ es el tensor de Ricci de la métrica $\mathrm{g}(\mathrm{t})$.

En el mismo artículo, Hamilton demostró el siguiente resultado

Teorema. Si $M^{3}$ es una variedad Riemanniana cerrada cuyo tensor de Ricci es definido positivo, entonces la variedad colapsa a un punto bajo el flujo de Ricci. Si se considera el flujo normalizado (volumen constante) converge a una variedad con curvatura constante positiva.

Entonces para demostrar la Conjetura de Poincaré es suficiente probar que toda 3-variedad cerrada simplemente conexa admite una estructura Riemanniana cuyo tensor de Ricci es definido positivo. 
En los últimos años, el matemático ruso Perelman ha usado el flujo de Ricci para demostrar la Conjetura de Geometrización, propuesta por el gran matemático Thruston, en la que confiere a cada pieza simple de cada 3-variedad una estructura Riemanniana. La Conjetura de Poincaré es consecuencia de la Conjetura de Geometrización. El trabajo de Perelman sobre el tema está contenido en tres artículos, [Pe1], [Pe2], [Pe3], disponibles a través de la red informática de comunicaciones. Sus resultados no han sido todavía publicados en una revista científica, pero son numerosos los matemáticos que han emitido una opinión positiva de su contenido, aunque también son muchos los que esperan cautelosamente su publicación. Esta prevención se justifica por la evolución de las anteriores pruebas anunciadas periódicamente, algunas de las cuales estuvieron vigentes bastante tiempo hasta que finalmente fueron desechadas por ser incompletas o erróneas.

Para mas información sobre el tema recomendamos los artículos recientes $[\mathrm{M}]$ y $[\mathrm{A}]$.

En la actualidad, podemos decir que el premio prometido por el Instituto Clay para este problema del milenio no ha sido todavía otorgado a ningún matemático. Por tanto debe ser considerado como un problema abierto.

\section{Referencias}

[A] M. T. Anderson, M.T. (2004) Geometrization of 3-manifolds via the Ricci flow. Notices Amer. Math. Soc. 51(2) 184-193.

[F] Freedman, M. (1982) The topology of four-dimensional manifolds. J. Differential Geom. 17, n ${ }^{\circ} 3,357-453$.

[H] Hamilton, R.S. (1982) Three-manifolds with positive Ricci curvature. J. Differential Geom. 17(2) 255-306.

[HLMW] Hilden, H.M., Lozano, M.T., Montesinos, J.M. y Whitten, W.C. On universal groups and three-manifolds. Invent. Math. 87(3) 441-456.

[M] Milnor, J. (2003) Towards the Poincaré conjecture and the classification of 3-manifolds. Notices Amer. Math. Soc. 50(10) 1226-1233.

[Pe1] Perelman, G. (2002) The entropy formula for the ricci flow and its geometric applications. Preprint math.DG/0211159.

[Pe2] Perelman, G. (2003) Finite extinction time for the solutions to the ricci flow on certain three-manifolds. Preprint math.DG/0307245.

[Pe3] Perelman, G. (2003). Ricci flow with surgery on three-manifold Preprint math.DG/0303109.

[Po1] Poincaré, H. (1900) Second complément a «l'analysis situs». Proc. London Math. Soc. 32, 277-308.

[Po2] Poincaré, H. (1904) Cinquieme complément a «'analysis situs». Rendiconti Circolo mat. Palermo, 18, 45-110. 
La conjetura de Poincaré. Un problema de topología

[Po3] Poincaré, H. (1921) Analyse de ses travaux scientifiques. Acta Math. 38, 36-135.

[Sm] Smale, S. (1961) Generalized Poincaré's conjecture in dimensions gre ater than tour. Ann. of Math. (2), 74, 391-406.

[St] Stallings, J.R. (1960) Polyhedral homotopy-spheres, Bull. Amer. Math. Soc., 66, 485-488.

[W] Wallace, A. (1960) Modifications and cobounding manifolds. Canad. J. Math. 12, 503-528. 